\title{
Computational Approach to Musical Consonance and Dissonance
}

\author{
Lluis L. Trulla ${ }^{1}$, Nicola Di Stefano ${ }^{2 *}$ and Alessandro Giuliani ${ }^{3}$ \\ ${ }^{1}$ Centre de Recerca Puig Rodó, Girona, Spain, ${ }^{2}$ Institute of Philosophy of Scientific and Technological Practice and \\ Laboratory of Developmental Neuroscience, Università Campus Bio-Medico di Roma, Rome, Italy, ${ }^{3}$ Environment and Health \\ Department, National Institute of Health, Rome, Italy
}

\section{OPEN ACCESS}

Edited by: Aleksey Nikolsky,

Independent Researcher, Los Angeles, CA, United States

Reviewed by: Juan G. Roederer, American Association of Retired

Persons, United States Susan Elizabeth Rogers, Berklee College of Music, United States

*Correspondence: Nicola Di Stefano n.distefano@unicampus.it

Specialty section: This article was submitted to Cognition,

a section of the journa

Frontiers in Psychology

Received: 28 August 2017 Accepted: 08 March 2018

Published: 04 April 2018

Citation:

Trulla LL, Di Stefano $N$ and Giuliani A (2018) Computational Approach to Musical Consonance and Dissonance.

Front. Psychol. 9:381. doi: 10.3389/fpsyg.2018.00381
In sixth century BC, Pythagoras discovered the mathematical foundation of musical consonance and dissonance. When auditory frequencies in small-integer ratios are combined, the result is a harmonious perception. In contrast, most frequency combinations result in audible, off-centered by-products labeled "beating" or "roughness;" these are reported by most listeners to sound dissonant. In this paper, we consider second-order beats, a kind of beating recognized as a product of neural processing, and demonstrate that the data-driven approach of Recurrence Quantification Analysis (RQA) allows for the reconstruction of the order in which interval ratios are ranked in music theory and harmony. We take advantage of computer-generated sounds containing all intervals over the span of an octave. To visualize second-order beats, we use a glissando from the unison to the octave. This procedure produces a profile of recurrence values that correspond to subsequent epochs along the original signal. We find that the higher recurrence peaks exactly match the epochs corresponding to just intonation frequency ratios. This result indicates a link between consonance and the dynamical features of the signal. Our findings integrate a new element into the existing theoretical models of consonance, thus providing a computational account of consonance in terms of dynamical systems theory. Finally, as it considers general features of acoustic signals, the present approach demonstrates a universal aspect of consonance and dissonance perception and provides a simple mathematical tool that could serve as a common framework for further neuro-psychological and music theory research.

Keywords: beating, recurrence quantification analysis, complex systems, non-linear signal analysis methods, Devil's staircase 


\section{INTRODUCTION}

Beating is the sensation that typically occurs when two sounds with similar frequencies mutually interfere, giving rise to a waveform with a rhythmic oscillation in amplitude. Following the fundamental contribution of Helmholtz's treatise, On the Sensation of Tone (1954), first published in 1863, contemporary explanations of consonance are grounded in the notions of beating and complex tones-i.e., sounds displaying a broad array of sinusoidal components (harmonics).

Roederer (2008, p. 35) provides an illuminating classification of the effects of superposing two pure tones depending on where in the listener's auditory system the sounds become entangled. The above mentioned beating is labeled by Roederer as "first-order beating," because it is processed mechanically in the cochlear fluid and along the basilar membrane. Evidence of the physiological basis of firstorder beating stems from the fact that its effect disappears when sounds are played separately in different ears-i.e., dichotically. Another kind of first-order beating effect is known as combination tones, which are produced by the non-linear interaction of waves in narrow spaces, such as the body of musical instruments or the inner ear. Combination tones can be considered as the product of two sine waves. A common example is the terzo suono theorized by Giuseppe Tartini (see Lohri, 2016). If $a$ and $b$ are two frequencies with $a>b$, then the terzo suono is a tone at frequency $a-b$ that is discernible only by the listener, because it is produced inside the inner ear rather than being caused by external air vibrations. Combination tones can be heard across the octave at sound pressure levels (SPLs) of $80 \mathrm{~dB}$ or higher, and across part of the octave at $50 \mathrm{~dB}$ SPL and above.

At $80 \mathrm{~dB}$ (or higher) while maintaining the interval around the octave, a distinct beating can be perceived. This disappears when $f_{2}=2 f_{1}$ (where $f_{1}$ and $f_{2}$ represent the two frequencies) and reappears as long as the octave becomes mistuned by a factor $\varepsilon$ (i.e., $f_{2}=2 f_{1}+\varepsilon$ ). The beating frequency turns out to be $\varepsilon$ (Plomp and Levelt, 1965). Beating "is created by the relatively quick changes produced by modulation frequencies in the region between about 15 to $300 \mathrm{~Hz}^{\prime}$ (Fastl and Zwicker, 2006, p. 257). Unlike first-order beats, the beating persists when tones are fed dichotically, implying that, in this case, beat perception is the result of neural processing. Hence, they are defined as "second-order beats" (Roederer, 2008). Secondorder beating shows a modulation in the vibration pattern, i.e., a periodic change in phase difference between the two sounds that form the interval (Roederer, 2008, p. 49), although no amplitude modulation is present. Second-order beats are also called "beats of mistuned consonances" because they are audible when pure tones are superposed to form a fifth (Plomp, 1976). In fact, whereas the vibration pattern of a correctly tuned fifth $\left(f_{2}=3 / 2 f_{1}\right)$ or fourth $\left(f_{2}=4 / 3 f_{1}\right)$ is static, the mistuned cases $f_{2}=3 / 2 f_{1}+\varepsilon$ and $f_{2}=4 / 3 f_{1}+\varepsilon$ cause the vibration pattern to change periodically in form, but not in amplitude. From the octave to the fifth and to the fourth, the second-order beats become faster (beating frequency being $\varepsilon$ for the octave, $2 \varepsilon$ for the fifth, and $3 \varepsilon$ for the fourth) as the vibration pattern grows in complexity (see Figure 1).

Their neural origin makes second-order beats an excellent phenomenon for investigating the link between the mathematical description of the signals and their neural processing, and consequently allows us to shed light on their perceived "pleasantness." To achieve a consistent picture of secondorder beats, it is fundamental to overcome the frequency-time space representation trade-off and the related problem of nonstationary signal characteristics.

Graphic representations of sound typically plot the course of amplitude over time or report the relative amplitudes of the different frequencies computed by the Fourier Transform. Thus, there is no mention of time in the latter, and no mention of frequency in the former. However, in the actual hearing process, time and frequency are strictly intermingled, because specific frequencies are processed at specific moments. This fact suggests that we should focus on the simultaneous analysis of time/frequency dimensions (Roads, 2001).

To determine the frequency of an oscillatory phenomenon, we must count the number $n$ of vibrations that occur within
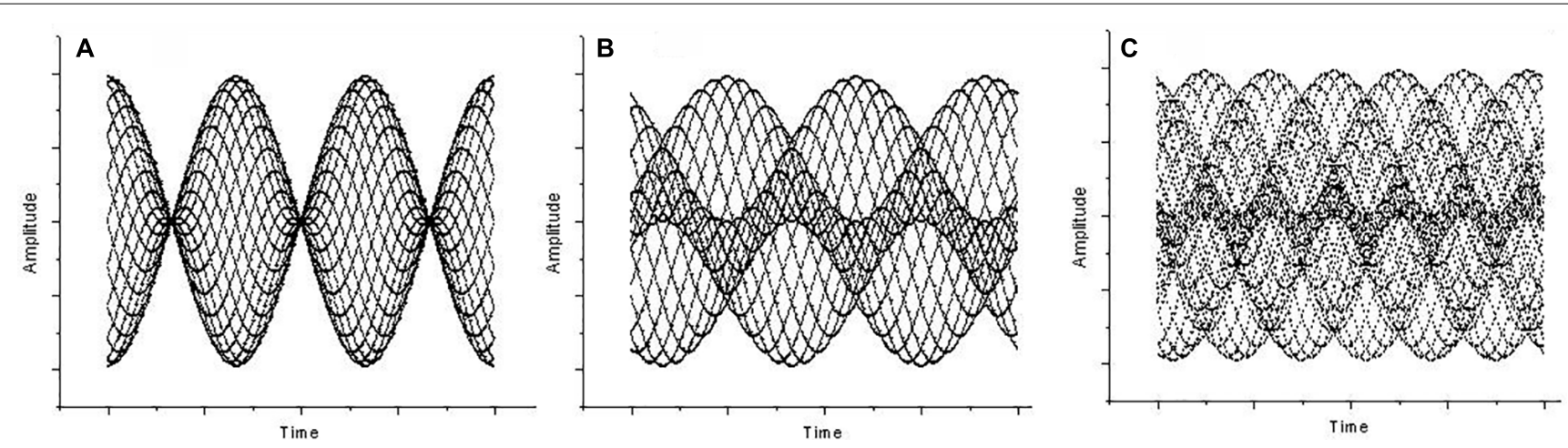

FIGURE 1 | Amplitude (y axis) against time ( $x$ axis) for (A) Mistuned unison, a case of first-order beating (the interval ratio is $400 / 403 \mathrm{~Hz}, \varepsilon=3,3$ beats). (B) Mistuned octave at $803 / 400 \mathrm{~Hz}(\varepsilon=3,3$ beats), a case of second-order beating. (C) Mistuned fifth at $603 / 400 \mathrm{~Hz}(\varepsilon=3$, the beats are $2 \varepsilon)$, also a case of second-order beating. 
a set time interval $\Lambda t$. As $n$ is an integer, the minimum error in measuring the frequency is one, thus generating a kind of uncertainty principle in the form $\Lambda f \geq 1 / \Lambda t$. Increasing the precision of the frequency reclaims a wider window in which to count the time, thus increasing the indetermination of the instant in which the specific frequency occurs.

It is possible to neglect the explicit consideration of time and visualize tone relationships within the octave by computing the ratio of two simultaneous frequencies and then plotting the interval ratio against the amplitude. This is achieved by forming a linear combination of two pure tone waves, a glissando from the unison $\left(f_{1}\right)$ to the octave $\left(2 f_{1}\right)$ and a firm wave at frequency $f_{1}$. Similar stimuli were previously adopted by Helmholtz (1954) and Kameoka and Kuriyagawa (1969a,b). More recently, Piana (2007) provided a phenomenological explanation of consonance and dissonance when moving from the glissando and ruling out intervals and harmonics. In the following, we propose a numerical approach to Helmholtz's glissando. Note that this approach maintains the time dimension in terms of the determined sequence of interactions between glissando and the fixed frequency. Focusing on these interactions allows us to overcome the trade-off in the frequency-time representation. For this purpose, we base our approach on the concept of recurrence, a simpler and more fundamental property of the signals with respect to the oscillation frequency (Eckmann et al., 1987; Marwan et al., 2007). The degree of recurrence of a series is estimated by the number of times a signal comes back to an already visited state (see section "Materials and Methods"), and can be computed by the application of recurrence quantification analysis (RQA) (Marwan et al., 2007). Estimating the recurrence rate avoids any stationarity assumption, as the estimate is obtained by a "computation window" sliding along the signal; the result is a profile of recurrence values relative to subsequent epochs along the original signal. This provides a model-free, discrete, and local estimation of the recurrent properties of the series, enabling a quantitative description of second-order beats. The recurrence peaks exactly match the values of the interval ratios corresponding to just intonation and are proportional to the order of consonance of the intervals, thus providing a link between consonance and the dynamical features of the signal.

\section{MATERIALS AND METHODS}

\section{Recurrence Quantification Analysis}

The original idea of describing non-stationary signals (which are not amenable to classical Fourier analysis) by means of recurrence dates back to the work of Ruelle's group (Eckmann et al., 1987). The authors introduced recurrence analysis as a purely graphical technique in the form of recurrence plots (RP). Webber and Zbilut (1994) then converted the RP approach into a quantitative technique (RQA) by defining some non-linear descriptors of the RP. RQA has been adopted for the assessment of time series structures in fields ranging from molecular dynamics to physiology and text analysis (Manetti et al., 1999; Orsucci et al., 2006; Marwan et al., 2007). In the field of music research, RQA has been successfully applied to song recognition (Serra et al., 2009) and in the definition of an objective basis of consonance of pure tones (Trulla et al., 2005). In general, this non-linear technique is especially useful for quantifying transient behavior far from the equilibrium (Trulla et al., 1996).

RQA builds upon the computation of a distance matrix between the rows (epochs) of the embedding matrix of the signal of interest, with the lag defined by the method of the first minimum of Mutual Information (Kennel et al., 1992). Given a scalar time series $\{x(i)=1 ; 2 ; 3 ; \ldots\}$, an embedding procedure generates a vector $\mathrm{Xi}=(\mathrm{x}(\mathrm{i}) ; \mathrm{x}(\mathrm{i}+L) ; \ldots$; $\mathrm{x}(\mathrm{i}+(m-1) L))$, where $m$ is the embedding dimension and $L$ is the lag. $\{\mathrm{Xi}=1 ; 2 ; 3 ; \ldots ; \mathrm{N}\}$ then represents the multi-dimensional process of the time series (signal) as a trajectory in $m$-dimensional space. RPs are symmetrical $\mathrm{N} \times \mathrm{N}$ matrices in which a point is placed at $(i ; j)$ whenever a point $X_{i}$ on the trajectory is close to another point $X_{j}$. The relative closeness between $X_{i}$ and $X_{j}$ is estimated by the Euclidian distance between these two vectors. If the distance falls below a threshold radius $(r)$, the two vectors (epochs, windows) are considered to be recurrent, and this is graphically indicated by a dot. The value of $r$ is usually set to $5-10 \%$ of the average pairwise distances between epochs. Therefore, RPs correspond to the symmetrical distance matrix between the epochs (rows of the embedding matrix) of the signal transformed into a binary $0 / 1$ matrix by the action of a threshold.

As an example, consider a time series $A$ made up of 10 consecutive values: $7,8,10,15,6,7,9,11,10,8$. To observe the recurrence structure of the series at the level of subsequent epochs of length 3 , we transform $A$ into the embedding matrix $A \mathrm{E}$ :

$\begin{array}{cccc}\mathrm{t} 0 & \mathrm{t}+1 & \mathrm{t}+2 & \text { epochs } \\ 7 & 8 & 10 & \text { ep1 } \\ 8 & 10 & 15 & \text { ep2 } \\ 10 & 15 & 6 & \text { ep3 } \\ 15 & 6 & 7 & \text { ep4 } \\ 6 & 7 & 9 & \text { ep5 } \\ 7 & 9 & 11 & \text { ep6 } \\ 9 & 11 & 10 & \text { ep7 } \\ 11 & 10 & 8 & \text { ep8 }\end{array}$

Thus, the original series has been projected into a threedimensional space in which the variables (columns) are the time-lagged original series and the statistical units (rows) are the overlapping epochs. The second step is to compute the Euclidean distances between the epochs. This generates the following distance matrix $\mathrm{AD}$ : 


$\begin{array}{cccc}\text { ep1 } & \text { ep2 } & \text { ep3 } & \text { ep4 } \\ 0 & & & \\ 5.477226 & 0 & & \\ 8.602325 & 10.48809 & 0 & 0 \\ 8.774964 & 11.35782 & 10.34408 & 9.273618 \\ \mathbf{1 . 7 3 2 0 5 1} & 7 & 9.433981 & 9.433981 \\ \mathbf{1 . 4 1 4 2 1} & 4.242641 & 8.3666 & 8.3666 \\ 3.605551 & 5.196152 & 5.744563 & 5.744563 \\ 4.898979 & 7.615773 & 5.477226 & \end{array}$

As the $\mathrm{AD}$ elements correspond to the Euclidean distances between corresponding epochs, the diagonal values are 0 , and the symmetric character of the distances implies the matrix can be written in lower-triangular form.

We now specify that two epochs are recurrent if their distance is less than $95 \%$ of all the between-epoch distances. The average value of the below-diagonal elements of $\mathrm{AD}$ is 6.48, and their standard deviation is 2.74. Thus, it is estimated that $95 \%$ of distances are greater than 1.74. This implies we have only two recurrences, corresponding to the epoch1-epoch5 and epoch1-epoch6 couples (bolded in the table).

Therefore, example series A has a recurrence rate of 0.071 (two recurrences out of 28 distinct distances) or, equivalently, a recurrence percentage equal to 7.1. The $\mathrm{AD}$ matrix corresponds to an RP with only two dots, at coordinates $(1,5)$ and $(1,6)$. Note that the recurrences can be identified without the need for any frequency estimation, thus resembling the hearing process that receives sounds as they occur in time.

To provide a quantitative measure of the recurrence, numerical RP descriptors were developed (Marwan et al., 2007). We now consider the proportion of recurrent points (dots) in a plot, called the recurrence. Going back to the music domain, Figure 2 reports the data relative to Figure 1 as RPs.

$\begin{array}{ccccc}\text { ep5 } & \text { ep6 } & \text { ep7 } & \text { ep8 } & \text { TIME } \\ & & & \text { ep1 } \\ & & \text { ep2 } \\ & & & \text { ep3 } \\ & & & \text { ep4 } \\ & & & \text { ep5 } \\ 0 & & & \text { ep6 } \\ 3 & 0 & & \text { ep7 } \\ 5.09902 & 3 & 0 & & \text { ep8 } \\ 5.91608 & 5.09902 & 3 & 0 & \end{array}$

\section{Software}

Files were generated using the sound editor Cool Edit Pro and saved in ASCII format before being fed to the Visual Recurrence Analysis (VRA) software. For the plots in Figure 1, we loaded a stereo file of 8000 samples/s to the audio editor, and sent a fixed pure tone of $400 \mathrm{~Hz}$ lasting $6 \mathrm{~s}$ through the left channel and a fixed pure tone of $403 \mathrm{~Hz}$ (Figure 1A), $803 \mathrm{~Hz}$ (Figure 1B), or $603 \mathrm{~Hz}$ (Figure 1C) for $6 \mathrm{~s}$ through the right channel. The sample type was then converted from stereo to mono. Figure 3 was generated by loading a stereo file of 8000 samples/s to the audio editor, and sending a linearly increasing sound from 360 to $840 \mathrm{~Hz}$ lasting $6 \mathrm{~s}$ to the left channel and a fixed pure tone of $400 \mathrm{~Hz}$ lasting $6 \mathrm{~s}$ to the right channel. Finally, the sample type was again converted from stereo to mono.

Figure 2 shows RPs for the data in Figure 1, i.e., $1 \mathrm{~s}(8000$ points) of a mistuned unison, octave, and fifth. The plots were generated by calculating the global recurrence using RQA, as there is no change along the sample. The recurrence of the data shown in Figure 3 was calculated using a windowing version of an $\mathrm{RP}$, whereby the recurrence is calculated repeatedly for a window that is continuously shifted along the whole sample. Among the RQA parameters, we chose the simplest one, Percent Recurrence, a descriptor that sets the percentage of recurrent points with respect to the non-trivial maximum [equal to $(\mathrm{N} \times(\mathrm{N}-1)) / 2$

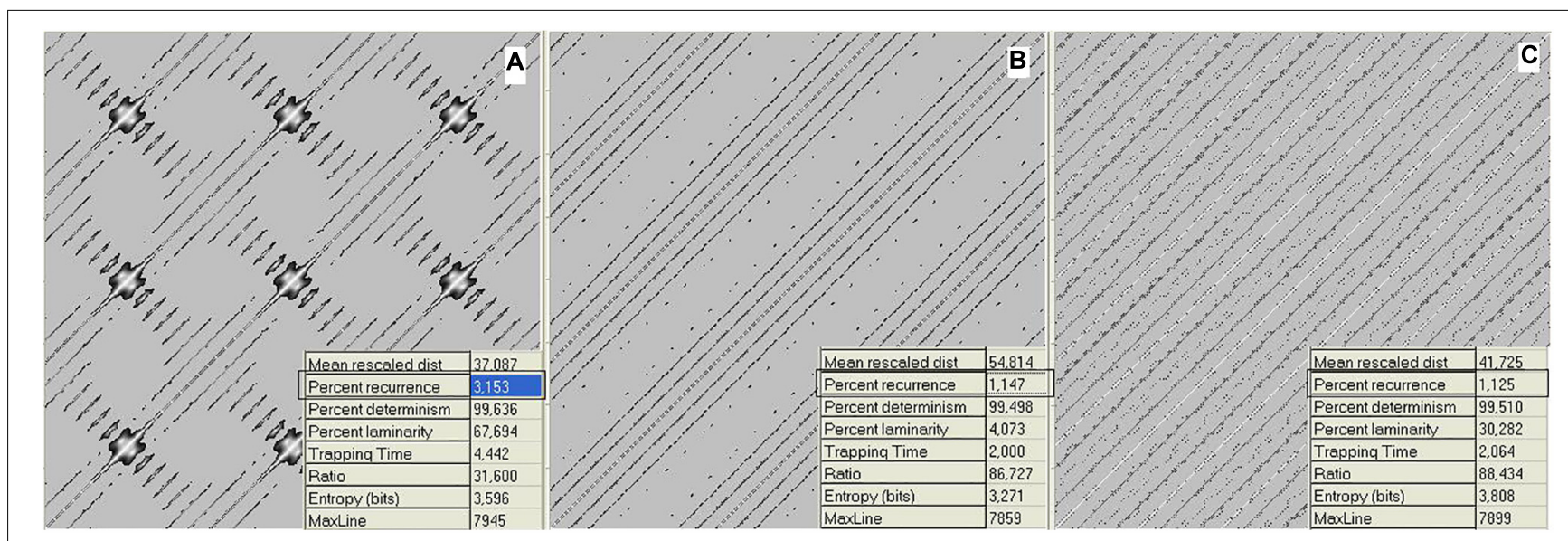

FIGURE 2 | Recurrence plots (RPs) of waveforms for mistuned unison (A), mistuned octave (B), and mistuned fifth (C). Calculations were performed on the data in Figure 1. Recurrence algorithm generates several descriptors (inset of the figures) of the recurrence distribution. Here, we consider the recurrence parameter. The axis refers to the discrete timing of the signal. RPs are graphical representations of a between-epochs distance matrix (see section "Materials and Methods"). The main diagonal line refers to the coincidence in time, while increasing distances (along both directions) correspond to the recurrences found at increasing delays. The individual dots denote the epoch pairs that have a distance value below the threshold and are thus considered to be recurrent. 


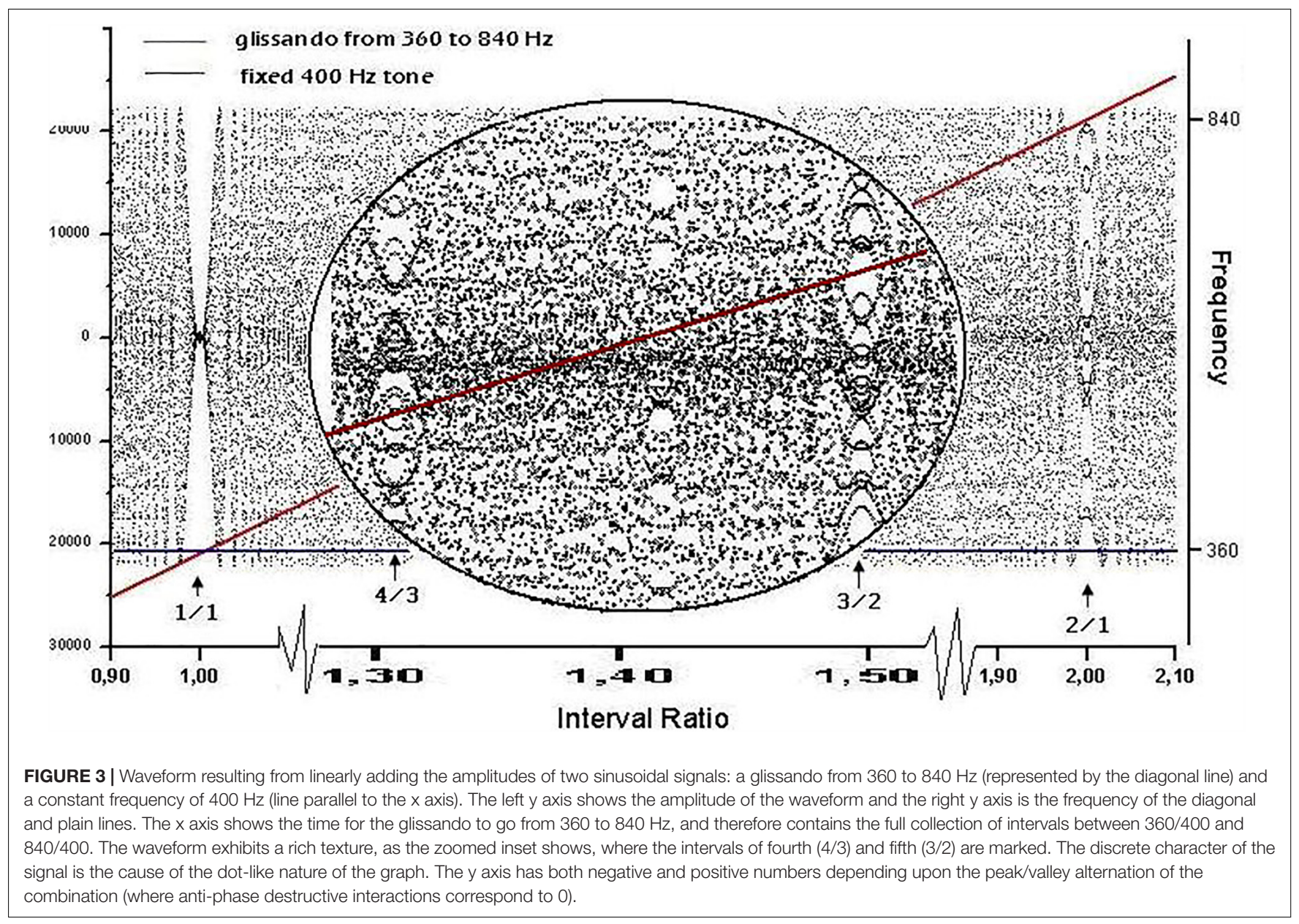

for an $\mathrm{N}$-point series]. The window for recurrence analysis was 480 points long and the shift was 48 points. The embedding dimension was 5 and the delay was 3 points.

MATLAB programs were obtained from http://sethares. engr.wisc.edu/consemi.html for Sethares' dissonance curve and http://courses.theophys.kth.se/5A1352/mfiles/devils.m for the theoretical Devil's staircase (see Discussion).

\section{RESULTS}

A non-stationary signal exploring all interval combinations within the octave can be generated by merging the course of two sounds into a single waveform. The first sound is set at constant frequency $f_{1}$ for the full duration of the course, while the second follows an ascending glissando from $f_{1}$ to $f_{2}=2 f_{1}$. Figure 3 shows an instance of the above procedure.

The most conspicuous singularity (recurrence peaks, see below) in the graph occurs when lines cross themselves, i.e., when $f_{2}=f_{1}$ (unison, interval ratio of 1:1). A second relevant case occurs at the interval ratio of 2:1, which corresponds to the octave. Less evident events occur at 3:2 (fifth) and 4:3 (fourth), as can be seen in the zoomed inset in Figure 3. Singularities in the waveform are thus localized where the frequency ratios are expressed by lower integers and with an apparent amplitude (or degree of singularity) matching the accepted ranking of consonance. In our representation, second-order beats appear as a zone of relative calm centered in rational numbers, surrounded by the tempestuous region of irrationals that Roederer (2008) called "beat holes."

Following the numerical solution of Helmholtz's glissando, we explore the glissando/constant frequency signal through an RQA windowing procedure called Recurrence Quantification of Epochs (RQE). RQE performs a scansion of the whole signal by sequentially selecting small windows-specifically episodes of 480 points-in which the RQA algorithm (with the consequent computation of recurrence rate for each episode) is applied. The subsequent windows are shifted by 48 points and the process is repeated throughout the entire file. For each iteration, we retain both the recurrence value and the interval ratio in which this value occurs, calculated as the mean of the interval ratios in the window. Figure 4 represents the degree of recurrence along the continuum of interval ratios within the octave.

Emergent features of the glissando are evident in Figure 4. Firstly, the higher peaks exactly correspond to the places of just intonation (see Trulla et al., 2005), thus establishing a link between pleasantness and the dynamical features (i.e., 


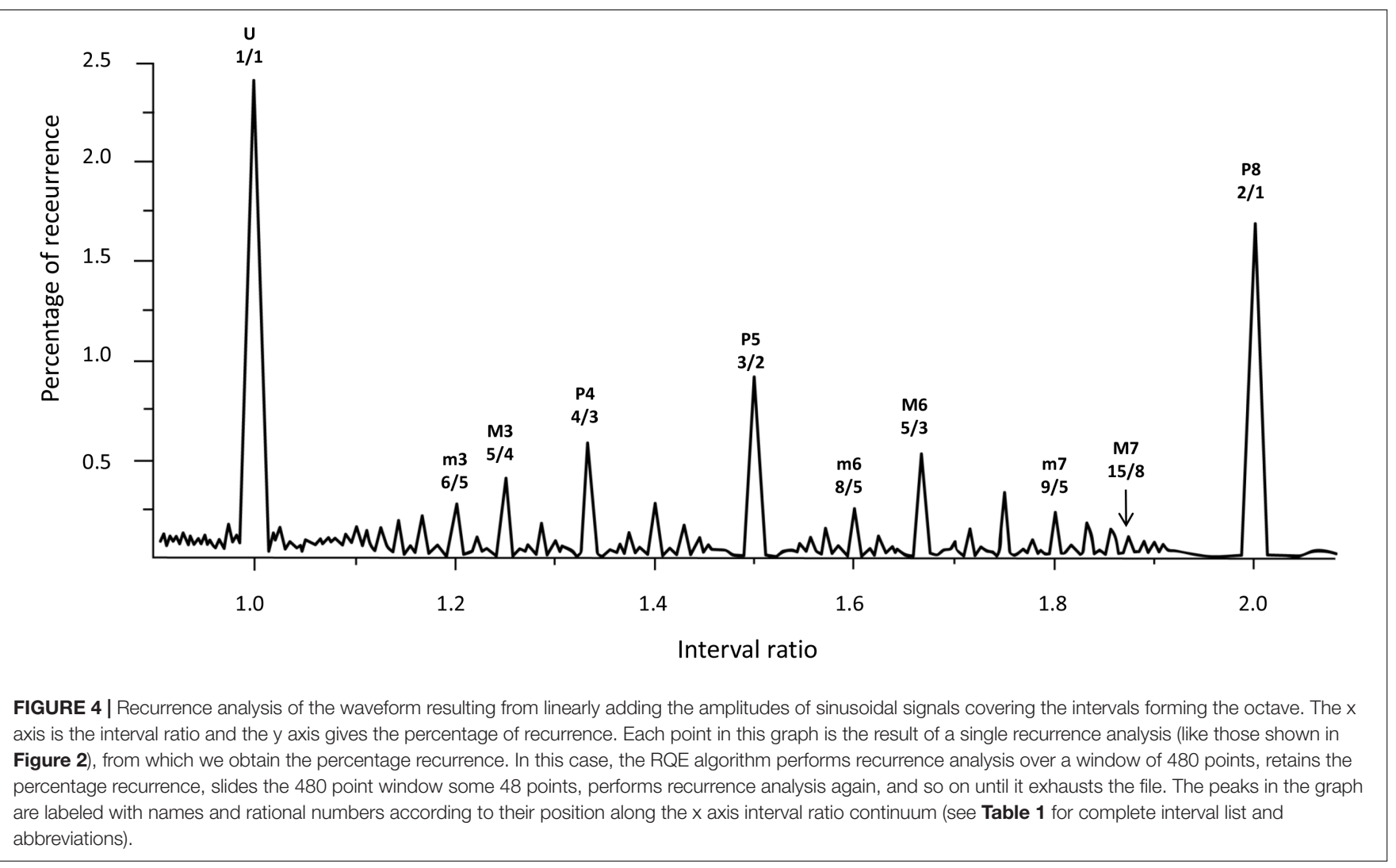

recurrence) of the signal. As expected from the numerical model, all peaks correspond to rational numbers. Secondly, it is worth noting the symmetry of the peaks around the perfect fifth. Moreover, the correlation between the extent of recurrence and the rank order of consonance derived from the literature evidences the link between the present model based on signal analysis and results from psychological approaches (see Schwartz et al., 2003, i.e., $\mathrm{U}>\mathrm{P} 8>\mathrm{P} 5>\mathrm{P} 4>\mathrm{M} 6>\mathrm{M} 3>\mathrm{m} 3>\mathrm{m} 6>\mathrm{m} 7>\mathrm{M} 7$, in decreasing order of consonance; see Table 1).

In summary, RQA allows us to establish a natural link between the signal properties and the consonance judgment of the listeners without any a priori hypothesis or frequency estimation. The reasons why integer numbers play such an important role in harmony has recently been addressed in the literature, with many different recipes presented for calculating the simplicity of the intervals. We use the consonance index provided by Frova (1999) to demonstrate the close relationship between the proposed recurrence index and the bare numerical characteristics of the intervals. If $m / n$ is the rational number in its lowest terms, Frova's index is $(m+n) /(m \times n)$ (Frova, 1999, p. 178). Figure 5 illustrates the correlation of this index with the notion of simplicity (i.e., degree of recurrence).

Whereas Frova's index is derived from the energy of the partials forming a complex sound, the percentage recurrence is a purely bottom-up phenomenological descriptor of a pure tone signal, relating recurrence (and consonance) to secondary beating and thus providing a natural (albeit roughly phenomenological) link between the signal properties and neural processing.

Note that the computation of recurrences gives very similar results with respect to models based on primary beating, such as the Plomp and Levelt model reported in Figure 6.

\section{DISCUSSION}

In this paragraph, we relate the self-similar appearance of the recurrence graph in Figure 4 to the mathematical fractal structures generated by physical processes. Figure 7 shows the empirical cumulative recurrence distribution (obtained by adding consecutive points) and a formal Devil's staircase in the $[1,2]$ interval: the similarities between the two graphs are remarkable.

The Devil's staircase pattern is a fingerprint of dynamical systems characterized by the mode-locking phenomenon (Schroeder, 1990, p. 171), which is crucially important in both music generation and perception. In the $17^{\text {th }}$ century, Christian Huygens studied mode-locking and discovered the phenomenon of resonance. He noticed that, after a time, the pendulums of two clocks fixed on the same mounting swung synchronously. The synchronization of two coupled oscillators starting from (slightly) different frequencies is called resonance. A more general case of resonant behavior appears when a specific constant frequency is periodically driven by an external power to oscillate at a different frequency; the so-called Devil's staircase 
pattern refers to the behavior of forced quasilinear oscillators. In the glissando, the constant frequency is the intrinsic frequency and the glissando the external periodic force. Every plateau in the Devil's staircase relates to a particular phase-locked solution (stable state), and its relative width forms a hierarchy that follows the explained propriety of rational numbers. The mathematical model for this case is the circle sine map (McCauley, 1994).

$$
\theta n+1=\theta n+\frac{p}{q}+\left(\frac{k}{2 \pi}\right) \sin (2 \pi \theta n)
$$

where $k$ is a coupling strength parameter that controls the degree of non-linearity. Without coupling $(k=0)$, the behavior of the system is expressed by the ratio $p / q$ (often called $\Omega$, the bare winding number). When $k>0$, the system locks into rational frequency ratios, preferably with small denominators. In this case, the long-term description of the system corresponds to $w$, the dressed winding number. For the critical value $k=1$, the infinite

TABLE 1 | Rank order of consonances and their degree of recurrence.

\begin{tabular}{|c|c|c|c|c|}
\hline Recurrence & $\begin{array}{l}\text { Interval } \\
\text { ratio }\end{array}$ & Label & Rational & Name \\
\hline 100,0 & 0.9999 & $\mathbf{U}$ & $1 / 1$ & Unison \\
\hline 89,1 & 2.0006 & P8 & $2 / 1$ & Octave \\
\hline 45,2 & 1.5003 & P5 & $3 / 2$ & Perfect fifth \\
\hline 30,6 & 1.3335 & P4 & $4 / 3$ & Perfect fourth \\
\hline 29,6 & 1.6671 & M6 & $5 / 3$ & Major sixth \\
\hline 23,4 & 1.2495 & M3 & $5 / 4$ & Major third \\
\hline 19,9 & 1.7499 & $\mathrm{H} 7$ & $7 / 4$ & Harmonic seventh \\
\hline 18,5 & 1.2003 & m3 & $6 / 5$ & Minor third \\
\hline 16,3 & 1.4007 & & $7 / 5$ & Septimal \\
\hline 15,4 & 1.5999 & $\mathrm{~m} 6$ & $8 / 5$ & Minor sixth \\
\hline 15,1 & 1.8003 & m7 & $9 / 5$ & Just minor seventh \\
\hline 14,2 & 1.1667 & & $7 / 6$ & Septimal minor third \\
\hline 11,9 & 1.2855 & & $9 / 7$ & Septimal major third \\
\hline 11,7 & 1.8339 & & $11 / 6$ & Undecimal neutral seventh \\
\hline 11,5 & 1.1427 & & $8 / 7$ & Septimal whole tone \\
\hline 10,1 & 1.4283 & & $10 / 7$ & Euler's tritone \\
\hline 9,8 & 1.1247 & Mt & $9 / 8$ & Major whole tone \\
\hline 9,7 & 1.7139 & & $12 / 7$ & Septimal major sixth \\
\hline 9,4 & 1.5711 & & $11 / 7$ & Undecimal augmented fifth \\
\hline 9,3 & 1.1115 & $\mathrm{mt}$ & $10 / 9$ & Minor whole tone \\
\hline 9,1 & 1.8567 & & $15 / 8$ & Classic major seventh \\
\hline 9,1 & 1.2219 & & $11 / 9$ & Undecimal neutral third \\
\hline 8,7 & 1.1006 & & $11 / 10$ & $4 / 5$ tone \\
\hline 8,6 & 1.3755 & & $11 / 8$ & $\begin{array}{l}\text { Undecimal semi-augmented } \\
\text { fourth }\end{array}$ \\
\hline 7,8 & 1.8747 & M7 & $15 / 8$ & Classic major seventh \\
\hline 7,7 & 1.2999 & & $13 / 10$ & $\begin{array}{l}\text { Tridecimal semi-diminished } \\
\text { fourth }\end{array}$ \\
\hline 7,6 & 1.6251 & & $13 / 8$ & Tridecimal neutral sixth \\
\hline 7,1 & 1.0911 & & $12 / 11$ & $3 / 4$ tone \\
\hline 6,8 & 1.8891 & & $17 / 9$ & Septendecimal minor third \\
\hline 6,8 & 1.1823 & & $13 / 11$ & Tridecimal minor third \\
\hline 6,7 & 1.4451 & D5 & $13 / 9$ & Tridecimal diminished fifth \\
\hline
\end{tabular}

Bolded values are intervals most used in Western harmony. number of locked frequency intervals corresponding to all the rational numbers between 0 and 1 cover the entire $\Omega$ range.

In our terms, $\Omega$ is the cumulative recurrence and $w$ is the interval ratio. In other words, the system is locked at any rational number-indicated as the interval ratio-but the width or extent of the lock comes from the cumulative recurrence. Thereby, most relevant consonances have extended areas around the lowest rationales-like the unison or octave-and a strong attraction exists toward these exact ratios. This is perfectly sound in terms of music theory.

The above considerations can be summarized in three main points:

(1) A purely empirical, data-driven analysis (RQA) has highlighted a fundamental property of signals (recurrence distribution) that matches the mathematical (number theory) and physical (mode-locking) theoretical background.

(2) The empirical results are consistent with both a theorydriven "simplicity index" (Frova's index) and with the order that music intervals are ranked in harmony.

(3) The focus on signal properties (second-order beatings) allows us to consider our results as a basis for modeling consonance and dissonance perception by combining data from both computational and cognitive models, e.g., based on artificial neural networks and Hebbian neuroplasticity (Pankovski and Pankovska, 2017).

Numerous studies have confirmed the adequacy of concepts from non-linear dynamics for music perception and construction (e.g., Cartwright et al., 2001, 2002, 2010), and for the study of synchronization among sound sources (Abel et al., 2009). Additionally, neuroscientific research has adopted non-linear dynamical models to describe phase-locked neural populations

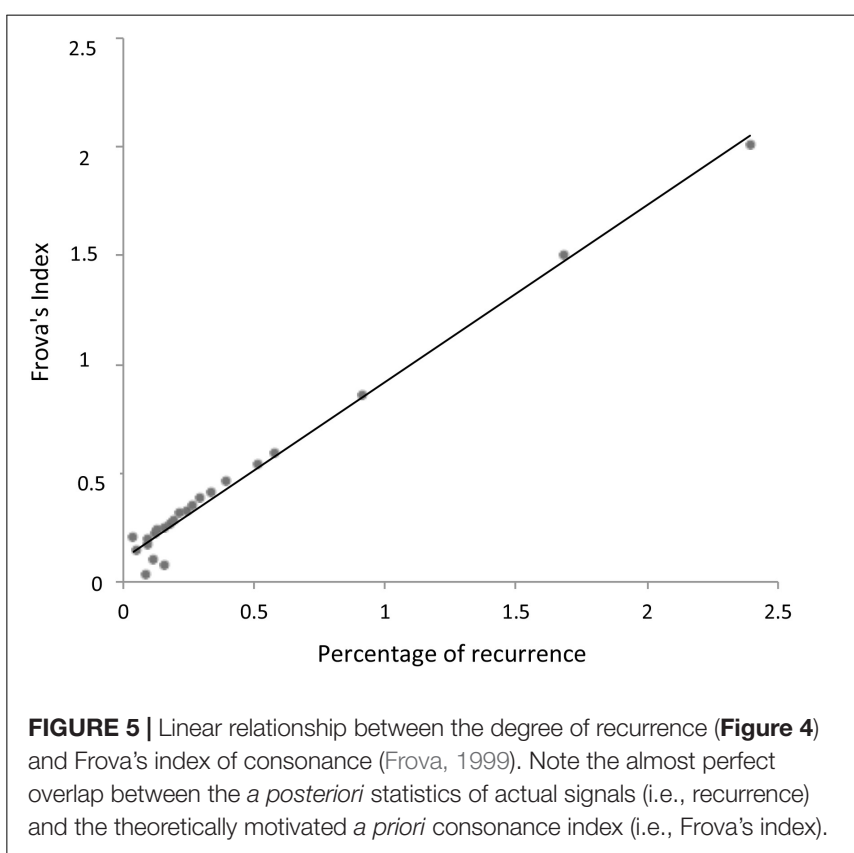




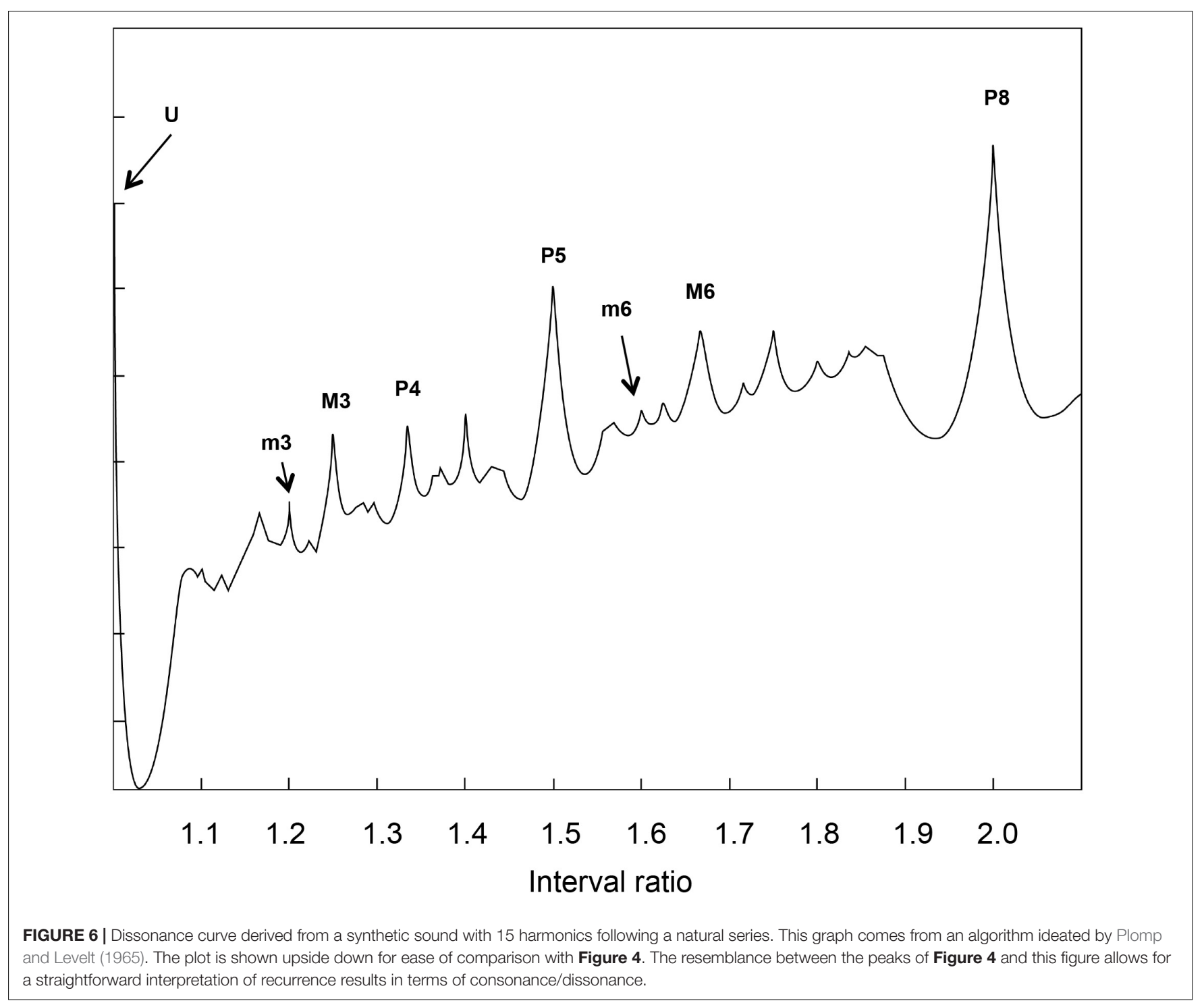

(Bidelman and Krishnan, 2009; Large and Almonte, 2012) and build in silico neuronal models (Lots and Stone, 2008).

Taken together, our work and previous results support the idea that the production and perception of sound are intimately linked, the perceived pleasantness of intervals being an intrinsic property of the signal (in terms of the degree of recurrence), and not only a secondary effect of the signal on the listener. In turn, this allows us to speculate on the auditory system. Second-order beats have been attributed to the central auditory nervous system, and neuronal webs are known to support phase-locking, as in the mammalian auditory system, in which neural activity in areas including the cochlear nucleus, inferior colliculus, and primary auditory cortex is phase-locked to the stimulus waveform (Large and Tretakis, 2005). The mode-locking model was proposed by Lots and Stone (2008) as the basis for musical consonance, leading to the development of a dynamical system model based on stylized neural oscillators producing both synchronization and mode-locking. These results support the idea that both parts of the communication system (the sender and the receiver of sounds) are similarly "wired." Bidelman and Heinz (2011) applied a waveform to a computational model of the acoustic nerve and, after deriving the autocorrelation function for the nerve fibers, generated the pitch salience profile for the different intervals, giving rise to a distribution that could be superimposed onto the recurrence rate (Figure 4). Using an artificial neural network model, Pankovski and Pankovska (2017) recently demonstrated that a specific auditory spectral distribution caused by non-linearities and Hebbian neuroplasticity are sufficient phenomena for a system to generate the consonance pattern.

In line with the literature on music perception (Benade, 1973; Roederer, 2008), we believe that the link between music generation and perception could rely on the fact that the vibrating elements of musical instruments undergo mode-locking into stationary complex vibration patterns. In turn, these can be recognized as the "best fit" to a harmonic template (resident in a properly wired neural circuit). Though this explanation 

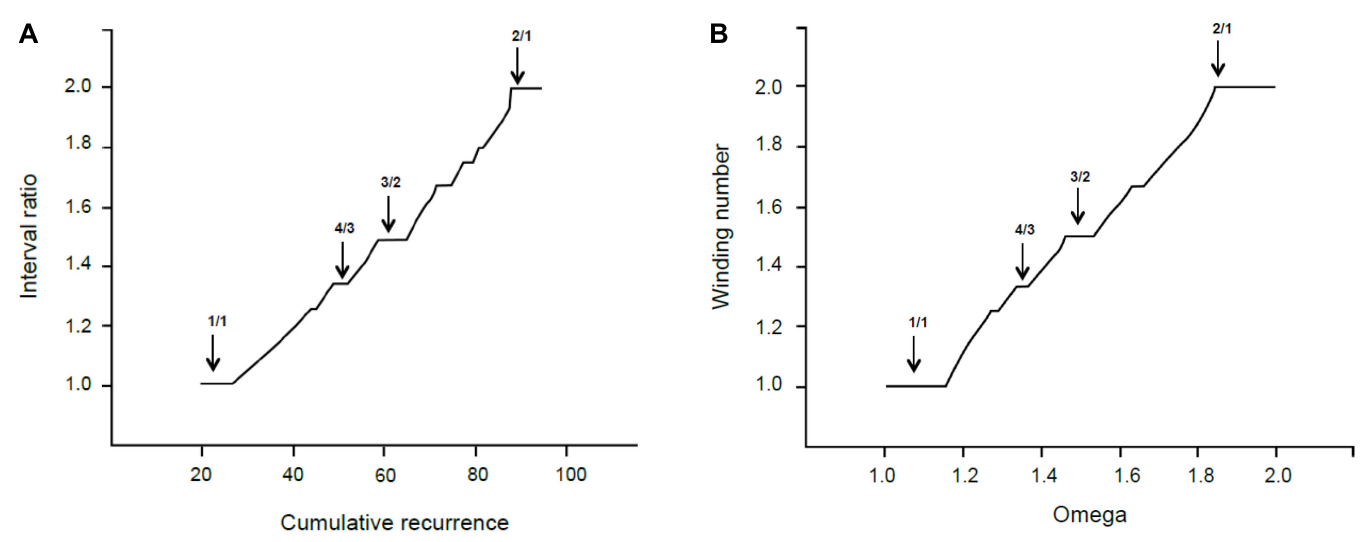

FIGURE 7 | (A) Interval ratio vs. cumulative recurrence. (B) Theoretical Devil's staircase from sine map with $k=1$.

stems from empirical correlations, we are convinced that the simplicity and versatility of the RQA approach could pave the way for neuro-psychological studies with the great advantage of considering the acoustic signal and the perceiver from the same mathematical perspective.

The origins of the distinction between consonance and dissonance have been hotly debated in recent years. As the phenomenon of consonance represents a key element of Western music theory, this has mainly been investigated in terms of Western science (i.e., mathematics, physics, psychoacoustics, and neuroscience). For this reason, Parncutt and Hair (2011) called for studies on the use of consonance and dissonance in non-Western cultures to be conducted in terms of local indigenous musicians, rather than in terms of Western science. In this direction, a relevant study published in Nature by McDermott et al. (2016) compares the harmonic preferences of people who have different degrees of exposure to Western music. An indigenous population from Bolivia (the Tsimané) was assumed to have no exposure to Western music, and their preferences were compared with groups of city residents in Bolivia and the United States with different degrees of exposure to Western music. The results show that the subjective preferences of Tsimané participants differ from those of the comparison groups; in particular, they failed to rate consonance as being more pleasant than dissonance. The authors state that, as the Tsimané are able to hear the acoustic distinctions associated with consonance and dissonance, the lack of a measurable preference for consonance appears to reflect difference in their aesthetic response to this contrast (McDermott et al., 2016, p. 549). Correctly, they state that the observed cross-cultural variation suggests that consonance preferences are unlikely to be innate, and so preference is probably acquired. However, the fact that the preference for consonance co-varies with presumptive exposure to Western culture is not sufficient to conclude that consonance perception is not biologically determined. Though preferences vary with cultures, the discrimination of consonance is a prerequisite for preference and has a biological basis, as supported by a large number of neurobiological studies (Koelsch and Mulder, 2002; Koelsch et al., 2005; Minati et al., 2008; Perani et al., 2010; Park et al., 2011; Wang, 2013). Investigating whether consonance perception is biologically determined or shaped by culture is likely to be misleading, as it conceives enculturation as a non-biologically constrained process. Harmonic intervals are a consequence of the entrainment of the nervous system with the sound excitation. This forms a universal biological foundation under any musical culture, determining the distinction between acoustic consonance and dissonance and leaving it to each culture to determine exactly how to employ these acoustic distinctions. However, the existence of different musical cultures and systems does not imply the lack of a shared natural/biological basis for music production. The interaction between nature and culture is much more complex, and cross-cultural variations in musical systems only show that biology does not rigidly determine music aesthetics. Similar considerations have led to a more adequate definition of music as a "biocultural phenomenon" (Cross, 2003).

\section{CONCLUSION}

The main contribution of this paper stems from the numerical solution of Helmholtz's glissando. Though the standard modern theory of consonance is based on first-order beating, we have shown that similar results can be obtained starting from secondorder beats. The recent interest in second-order beating has been fruitful for models of pitch recognition or neural circuitry (see Roederer, 2008), but not for theories on consonance.

Scholars have started to consider music from the perspective of dynamical systems, both in neurobiological and physical terms, showing that mode-locking models can explain how the nervous system manages sound and is engaged in the ranking of consonances. The resemblance between the formal Devil's staircase model and the cumulative recurrence distribution strengthens this idea.

From a methodological perspective, the main contribution of this work is to provide neuroscience scholars with an extremely simple and model-free tool (RQA) that approaches the acoustic signal and the listener's perception system with the same mathematical method. Different RQA applications have 
been reported in research on otoacoustic emission (see, for example, Zimatore et al., 2002, 2003). We are therefore confident that the use of a simple statistical approach will foster interactions between music theory and neuro-psychological approaches.

Finally, our results support the idea of natural roots of consonance perception, and are thus in line with several studies published in recent years (see, for example, Wang, 2013; Bowling and Purves, 2015; Nikolsky, 2015; Foo et al., 2016; GonzálezGarcía et al., 2016; Di Stefano et al., 2017). However, as proved by McDermott et al. (2016), the role of perception in the formulation of aesthetic judgment remains unclear. Therefore, musical consonance and dissonance remains a hotly debated topic (see Bowling et al., 2017), in need of further research to merge different approaches into a consistent theory.

\section{AUTHOR CONTRIBUTIONS}

LT originally conceived the idea of the paper, elaborated the stimuli, provided all the figures, and significantly contributed

\section{REFERENCES}

Abel, M., Ahnert, K., and Bergweiler, S. (2009). Synchronization of sound sources. Phys. Rev. Lett. 103:114301. doi: 10.1103/PhysRevLett.103.114301

Benade, A. H. (1973). The physics of brasses. Sci. Am. 229, 24-35. doi: 10.1038/ scientificamerican0773-24

Bidelman, G. M., and Heinz, M. G. (2011). Auditory-nerve responses predict pitch attributes related to musical consonance-dissonance for normal and impaired hearing. J. Acoust. Soc. Am. 130, 1488-1502. doi: 10.1121/1.360 5559

Bidelman, G. M., and Krishnan, A. (2009). Neural correlates of consonance, dissonance, and the hierarchy of musical pitch in the human brainstem. J. Neurosci. 29, 13165-13171. doi: 10.1523/JNEUROSCI.3900-09.2009

Bowling, D. L., Hoeschele, M., Kamraan, Z. G., and Tecumseh Fitch, W. (2017). The nature and nurture of musical consonance. Music Percept. 35, 118-121. doi: $10.1525 / \mathrm{mp} \cdot 2017.35 .1 .118$

Bowling, D. L., and Purves, D. (2015). A biological rationale for musical consonance. Proc. Natl. Acad. Sci. U.S.A. 112, 11155-11160. doi: 10.1073/pnas. 1505768112

Cartwright, J. H. E., Douthettb, J., González, D. L., Krantzd, R., and Piro, O. (2010). Two musical paths to the Farey series and devil's staircase. J. Math. Music 4, 57-74. doi: 10.1080/17459737.2010.485001

Cartwright, J. H. E., Gonzalez, D. L., and Piro, O. (2001). Pitch perception: a dynamical-systems perspective. Proc. Natl. Acad. Sci. U.S.A. 98, 4855-4859. doi: $10.1073 /$ pnas. 081070998

Cartwright, J. H. E., Gonzalez, D. L., Piro, O., and Stanziali, D. (2002). Aesthetics, dynamics, and musical scales: a golden connection. J. New Music Res. 31, 51-58. doi: 10.1076/jnmr.31.1.51.8099

Cross, I. (2003). Music as a biocultural phenomenon. Ann. N. Y. Acad. Sci. 999, 106-111. doi: 10.1196/annals. 1284.010

Di Stefano, N., Focaroli, V., Giuliani, A., Formica, D., Taffoni, F., and Keller, F. (2017). A new research method to test auditory preferences in young listeners: results from a consonance versus dissonance perception study. Psychol. Music 45, 699-712. doi: 10.1177/0305735616681205

Eckmann, J. P., Kamphorst, S. O., and Ruelle, D. (1987). Recurrence plots of dynamical systems. Europhys. Lett. 4, 973-976. doi: 10.1209/0295-5075/4/9/004 Fastl, H., and Zwicker, E. (2006). Psychoacoustic. Facts and Models. Berlin: Springer.

Foo, F., King-Stephens, D., Weber, P., Laxer, K., Parvizi, J., and Knight, R. T. (2016). Differential processing of consonance and dissonance within the human superior temporal gyrus. Front. Hum. Neurosci. 10:154. doi: 10.3389/fnhum. 2016.00154

Frova, A. (1999). Fisica nella Musica. Bologna: Zanichelli. to the results and discussion. NDS prepared the manuscript, co-authored the introduction and the results with LT, contributed to the discussion, wrote the conclusion, and finally revised the entire draft. AG wrote the section "Recurrence Quantification Analysis," reviewed the entire manuscript, and suggested useful ideas for the discussion. All authors equally contributed to the revision of the manuscript before agreeing on the final version.

\section{FUNDING}

This work was funded by the Institute of Philosophy of Scientific and Technological Practice, Campus Bio-Medico University of Rome, under a 2015 Grant on "Embodiment."

\section{ACKNOWLEDGMENTS}

LT is grateful to Universitat Pompeu Fabra (UPF) of Barcelona for access to their library.

González-García, N., González, M. A., and Rendón, P. L. (2016). Neural activity related to discrimination and vocal production of consonant and dissonant musical intervals. Brain Res. 1643, 59-69. doi: 10.1016/j.brainres.2016.04.065

Helmholtz, H. (1954). On the Sensations of Tone. New York, NY: Dover Publications.

Kameoka, A., and Kuriyagawa, M. (1969a). Consonance theory part I: consonance of dyads. J. Acoust. Soc. Am. 45, 1451-1459. doi: 10.1121/1.1911623

Kameoka, A., and Kuriyagawa, M. (1969b). Consonance theory part II: consonance of complex tones and its calculation method. J. Acoust. Soc. Am. 45, 1460-1469. doi: 10.1121/1.1911624

Kennel, M. B., Brown, R., and Abarbanel, H. D. (1992). Determining embedding dimension for phase-space reconstruction using a geometrical construction. Phys. Rev. A 45, 3403. doi: 10.1103/PhysRevA.45.3403

Koelsch, S., Fritz, T., Schulze, K., Alsop, D., and Schlaug, G. (2005). Adults and children processing music: An fMRI study. Neuroimage 25, 1068-1076. doi: 10.1016/j.neuroimage.2004.12.050

Koelsch, S., and Mulder, J. (2002). Electric brain responses to inappropriate harmonies during listening to expressive music. Clin. Neurophysiol. 113, 862-869. doi: 10.1016/S1388-2457(02)00050-0

Large, E. W., and Almonte, F. V. (2012). Neurodynamics, tonality, and the auditory brainstem response. Ann. N. Y. Acad. Sci. 1252, E1-E7. doi: 10.1111/j.17496632.2012.06594.x

Large, E. W., and Tretakis, A. E. (2005). Tonality and nonlinear resonance. Ann. N. Y. Acad. Sci. 1060, 53-56. doi: 10.1196/annals.1360.046

Lohri, A. (2016). Kombinationstöne und Tartinis "terzo suono". Mainz: Schott Music.

Lots, I. S., and Stone, L. (2008). Perception of musical consonance and dissonance: an outcome of neural synchronization. J. R. Soc. Interface 5, 1429-1434. doi: 10.1098/rsif.2008.0143

Manetti, C., Ceruso, M. A., Giuliani, A., Webber, C. L. Jr., and Zbilut, J. P. (1999). Recurrence quantification analysis as a tool for characterization of molecular dynamics simulations. Phys. Rev. E 59, 992-998. doi: 10.1103/PhysRevE.59.992

Marwan, N., Romano, M. C., Thiel, M., and Kurths, J. (2007). Recurrence plots for the analysis of complex systems. Phys. Rep. 438, 237-329. doi: 10.1016/j. physrep.2006.11.001

McCauley, J. L. (1994). Chaos, Dynamics and Fractals. Cambridge: Cambridge University Press.

McDermott, J., Schultz, A. F., Undurraga, E. A., and Godoy, R. A. (2016). Indifference to dissonance in native Amazonians reveals cultural variations in music perception. Nature 535, 547-550. doi: 10.1038/nature18635

Minati, L., Rosazza, C., D’Incerti, L., Pietrocini, E., Valentini, L., Scaioli, V., et al. (2008). FMRI/ERP of musical syntax: comparison of melodies and 
unstructured note sequences. Neuroreport 19, 1381-1385. doi: 10.1097/WNR. 0b013e32830c694b

Nikolsky, A. (2015). Evolution of tonal organization in music mirrors symbolic representation of perceptual reality. Part-1: prehistoric. Front. Psychol. 6:1405. doi: 10.3389/fpsyg.2015.01405

Orsucci, F., Giuliani, A., Webber, C., Zbilut, J., Fonagy, P., and Mazza, M. (2006). Combinatorics and synchronization in natural semiotics. Phys. A Stat. Mech. Appl. 361, 665-676. doi: 10.1016/j.physa.2005.06.044

Pankovski, T., and Pankovska, E. (2017). Emergence of the consonance pattern within synaptic weights of a neural network featuring Hebbian neuroplasticity. Biol. Insp. Cong. Arch. 22, 82-94. doi: 10.1016/j.bica.2017.09.001

Park, J. Y., Park, H., Kim, J., and Park, H. J. (2011). Consonant chords stimulate higher EEG gamma activity than dissonant chords. Neurosci. Lett. 488, 101-105. doi: 10.1016/j.neulet.2010.11.011

Parncutt, R., and Hair, G. (2011). Consonance and dissonance in theory and psychology: disentangling dissonant dichotomies. J. Interdiscip. Music Stud. 5, 119-166.

Perani, D., Saccuman, M. C., Scifo, P., Spada, D., Andreolli, G., Rovelli, R., et al. (2010). Functional specializations for music processing in the human newborn brain. Proc. Natl. Acad. Sci. U.S.A. 107, 4758-4763. doi: 10.1073/pnas. 0909074107

Piana, G. (2007). Barlumi per una Filosofia Della Musica. Morrisville: Lulu Press.

Plomp, R. (1976). Aspects of Tone Sensation: A Psychophysical Study. London: Academic Press.

Plomp, R., and Levelt, W. J. M. (1965). Tonal consonance and critical bandwidth. J. Acoust. Soc. Am. 38, 548-560. doi: 10.1121/1.1909741

Roads, C. (2001). Microsound. Cambridge, MA: The MIT Press.

Roederer, J. G. (2008). The Physics and Psychophysics of Music. New York, NY: Springer.

Schroeder, M. (1990). Fractals, Chaos, Power Laws. New York, NY: W.H Freeman and Co.

Schwartz, D. A., Howe, C. Q., and Purves, D. (2003). The statistical structure of human speech sounds predicts music universals. J. Neurosci. 23, 7160-7168.
Serra, J., Serra, X., and Andrzejak, R. G. (2009). Cross recurrence quantification for cover song identification. New J. Phys. 11:093017. doi: 10.1088/1367-2630/11/ 9/093017

Trulla, L. L., Giuliani, A., Zbilut, J. P., and Webber, C. L. (1996). Recurrence quantification analysis of the logistic equation with transients. Phys. Lett. A 223, 255-260. doi: 10.1016/S0375-9601(96)00741-4

Trulla, L. L., Giuliani, A., Zimatore, G., Colosimo, A., and Zbilut, J. P. (2005). Non-linear assessment of musical consonance. Electron. J. Theor. Phys. 8, 22-34.

Wang, X. (2013). The harmonic organization of auditory cortex. Front. Syst. Neurosci. 7:114. doi: 10.3389/fnsys.2013.00114

Webber, C. L., and Zbilut, J. P. (1994). Dynamical assessment of physiological systems and states using recurrence plot strategies. J. Appl. Physiol. 76, 965-973. doi: 10.1152/jappl.1994.76.2.965

Zimatore, G., Giuliani, A., Hatzopoulos, S., Martini, A., and Colosimo, A. (2003). Otoacoustic emissions at different click intensities: invariant and subjectdependent features. J. Appl. Physiol. 95, 2299-2305. doi: 10.1152/japplphysiol. 00667.2003

Zimatore, G., Hatzopoulos, S., Giuliani, A., Martini, A., and Colosimo, A. (2002). Comparison of transient otoacoustic emission (TEOAE) responses from neonatal and adult ears. J. Appl. Physiol. 92, 2521-2528. doi: 10.1152/ japplphysiol.01163.2001

Conflict of Interest Statement: The authors declare that the research was conducted in the absence of any commercial or financial relationships that could be construed as a potential conflict of interest.

Copyright (C) 2018 Trulla, Di Stefano and Giuliani. This is an open-access article distributed under the terms of the Creative Commons Attribution License (CC BY). The use, distribution or reproduction in other forums is permitted, provided the original author(s) and the copyright owner are credited and that the original publication in this journal is cited, in accordance with accepted academic practice. No use, distribution or reproduction is permitted which does not comply with these terms. 